\title{
Labyrinthe
}

28 | 2007 (3)

Des Juifs contre l'émancipation

\section{L'émancipation à « marche forcée » : les Juifs d'Algérie et le décret Crémieux}

David Nadjari

\section{OpenEdition}

Journals

Édition électronique

URL : https://journals.openedition.org/labyrinthe/2893

DOI : $10.4000 /$ labyrinthe.2893

ISSN : 1950-6031

Éditeur

Hermann

Édition imprimée

Date de publication : 21 décembre 2007

Pagination : 77-89

ISBN : 978-2-9526131-5-6

Référence électronique

David Nadjari, «L'émancipation à « marche forcée » : les Juifs d'Algérie et le décret Crémieux »,

Labyrinthe [En ligne], 28 | 2007 (3), mis en ligne le 01 octobre 2009, consulté le 21 septembre 2021.

URL : http://journals.openedition.org/labyrinthe/2893; DOI : https://doi.org/10.4000/labyrinthe.2893

Propriété intellectuelle 


\title{
L'ÉMANCIPATION À «MARCHE FORCÉE»: les Juifs d'Algérie et le décret Crémieux
}

\author{
David NADJARI \\ david.nadjari@wanadoo.fr
}

Cet article se propose d'étudier un cas spécifique d'application du projet émancipateur français: celui des Juifs d'Algérie.

C'est en 1870, à la faveur du décret Crémieux, que ceux-ci entrent de plain-pied dans la citoyenneté française. Rétrospectivement, en particulier à la lumière du «rapatriement» des pieds-noirs de 1962, cet acte politique a tout l'air d'une évidence: il ne s'agissait là que d'une «normalisation» de la situation des israélites «d'outre-mer» résidant désormais sur un territoire devenu français après la conquête militaire de 1830. La III ${ }^{\text {e }}$ République naissante n'avait fait là que parachever le grand dessein que la France révolutionnaire et napoléonienne avait conçu pour «ses Juifs». Ce mythe de la normalisation et de la continuité bute cependant sur les faits. En dépit d'un certain nombre de similitudes, le processus qui a abouti à la naturalisation collective des Juifs d'Algérie présente des différences majeures avec celui qui a débouché sur l'émancipation politique des Juifs de métropole, plusieurs décennies auparavant.

La plus notable est l'origine extra-européenne des communautés algériennes au départ. Contrairement à celles d'Europe, elles ont été façonnées par une cohabitation pluriséculaire avec l'Islam et présentent un décalage culturel fondamental avec la France des Lumières. La «régénération » que l'émancipation pose en préalable ne se conçoit pas dès lors avec la même puissance dans les deux cas.

La deuxième différence tient au contexte colonial. L'État émancipateur devenu État colonisateur ne présente pas exactement les mêmes logiques d'action. Ainsi, la réforme des institutions du judaïsme algérien a répondu - du moins au départ - aussi bien aux impératifs de la 
domination politique d'une population «indigène» qu'à l'idéal universaliste de diffusion des Lumières.

L'étude du cas algérien apparaît bien comme un modèle de ce que Pierre Birnbaum ${ }^{1}$ appelle un «processus d'étatisation révolutionnaire». Comme pour les Juifs de France, l'attitude de l'État vis-à-vis des Juifs d'Algérie fut une démarche autoritaire. Mais le contexte ethnique, culturel et politique ne pouvait que rendre la tâche plus difficile et les résistances au projet de «régénération» plus fortes.

Nous voudrions ici retracer d'abord les étapes qui ont conduit à l'octroi de la citoyenneté française aux Juifs d'Algérie; puis analyser les réformes politiques et institutionnelles que ce processus a amenées; avant de poser la question des réactions des Juifs algériens au démantèlement de leur cadre communautaire traditionnel.

\section{«Ils sont d'un fanatisme outré et persécuteur!»}

Avant de s'intéresser à l'action des autorités françaises, rappelons rapidement ce qu'il en est des institutions juives algériennes à la veille de la conquête française pour mesurer ensuite le bouleversement opéré par le colonisateur.

À la veille de la conquête de 1830, il n'est pas exagéré d'affirmer que le judaïsme algérien est aussi éloigné culturellement de la France des Lumières que l'Islam. Trois traits caractérisent cette distance.

Le premier est d'ordre civilisationnel. Le judaïsme algérien, et plus largement le judaïsme maghrébin est un judaïsme traditionnel qui fonde principalement son ordre social et religieux non seulement sur la loi de Dieu et sur l'enseignement rabbinique, mais aussi sur un socle de valeurs, de croyances et de pratiques communes à toutes les ethnies nord-africaines. La cohabitation pluriséculaire avec l'Islam a enfanté une culture originale dont la langue (le judéo-arabe), les rituels de fertilité ou la pratique du maraboutisme sont les témoins.

La deuxième caractéristique est d'ordre institutionnel. La soumission au souverain musulman a une conséquence majeure sur l'organisation communautaire: son indépendance. En effet, le pacte de la dhimma,

1. Pierre Birnbaum (dir.), Histoire politique des Juifs en France : entre universalisme et particularisme, Paris, Presses de la Fondation nationale des sciences politiques, 1990. 
qui confère aux Juifs le statut d'inférieur-protégé, accorde - outre ses aspects très contraignants - une liberté presque totale aux communautés dans la gestion de leur culte. Chacune d'entre elles (et indépendamment les unes des autres) est libre de gérer ses ressources, de nommer ses rabbins, de tenir un état civil, d'entretenir les infrastructures cultuelles (synagogues, bains rituels, cimetières).

Enfin, le troisième élément déterminant est d'ordre social et politique. De l'indépendance communautaire et de l'extrême pauvreté, résulte une organisation clanique de la société juive. La nécessité d'être représenté auprès du souverain musulman stimule la compétition entre les grandes familles et le clientélisme. Cet aspect est tout à fait essentiel pour expliquer qu'à la veille de l'arrivée des Français l'espace public communautaire est quasi inexistant et que la gestion du culte a été entièrement privatisée par les chefs de clan.

La conquête militaire de l'Algérie par la France eut d'importantes conséquences sur cette organisation communautaire. L'action du colonisateur fut d'abord dictée par les impératifs militaires et politiques de la conquête. Quelle que fut la sympathie des Juifs algériens vis-à-vis du conquérant, il s'agissait en premier lieu de soumettre les populations «indigènes». À ce titre, les Juifs ne faisaient pas exception.

En 1831, les muqaddam, représentants traditionnels de la nation juive auprès du souverain musulman, sont placés sous autorité militaire, avant d'être supprimés, en 1836. En 1842, c'est au tour des tribunaux rabbiniques d'être dissous. L'opacité de l'activité du beit-din constituait un frein à la surveillance française et l'on décida de transférer toutes ses attributions aux juridictions françaises. Celles-ci avaient alors à se prononcer selon le droit français pour toutes les affaires de droit commun et selon la loi hébraïque pour le statut personnel. Les ex-dayanim étaient quant à eux relégués au rang de simples consultants. Ce statut mixte portait évidemment en germe une multitude d'incohérences et fut donc l'objet de nombreux ajustements, qui allèrent toujours dans le sens d'une restriction des cas d'application de la loi religieuse.

Au total, en une grosse décennie, la plupart des institutions corporatives juives furent soit placées sous tutelle, soit démantelées et remplacées par celles du conquérant.

Déterminer le rôle des Juifs de France est capital pour comprendre la tournure que prirent dès lors les événements. Dès les premiers temps de la conquête, les Juifs de France s'étaient préoccupés du sort des Juifs 
de l'Algérie occupée. Leur méconnaissance de l'univers juif maghrébin était alors totale, dans la mesure où les relations entre les deux communautés avaient été quasiment nulles jusque-là. L'ignorance faisait naître en France une sorte d'imagerie romantique d'un peuple «menant encore la vie guerrière et nomade des anciens patriarches ${ }^{1} »$. Cela dit, l'émotion était réelle de voir enfin libérée une nation qu'ils croyaient opprimée par les Turcs. Suivant une rhétorique biblique, ils se félicitaient de la générosité de la France d'avoir affranchi les Juifs du joug ottoman.

Les premières démarches des autorités consistoriales françaises avaient ainsi une dimension principalement humanitaire. Dès 1833, elles réclamèrent la création d'un consistoire à Alger. Mais cette demande ne faisait que suivre celle de l'Église catholique d'implanter un évêché dans la colonie et n'avait par conséquent que peu de signification. En 1837, on trouve une nouvelle formulation de la requête mais celle-ci ne fut pas suivie d'effet. Placés jusqu'alors sous le signe de la charité, l'action et le discours des israélites français connurent quelques temps plus tard, en 1840, un premier soubresaut. Cette année-là, une lettre envoyée par un médecin israélite de l'armée d'Afrique parvint au consistoire central. Son auteur, le docteur Goldsheider, voulait y exprimer son témoignage sur la réalité des Juifs indigènes qu'il avait rencontrée. Le texte qui fut par la suite publié dans les revues israélites françaises avait en effet de quoi surprendre:

C'est une race exécrable, fourbe, avide. Ils joignent toute la bassesse de l'esclavage aux vices les plus dépravés [...]. Ils sont d'un fanatisme outré et persécuteur [...]. Ils parlent l'arabe mais paraît-il qu'ils le prononcent autrement que les Maures, et ils écrivent l'arabe avec des lettres hébraïques qui [ne] sont pas les mêmes que chez nous. Je suis allé aussi dans plusieurs synagogues qui sont très nombreuses; les écuries en France sont plus propres que leurs temples [...]. Aussitôt que paraît le sefer [le rouleau de la Torah], ils se jettent dessus et font mille simagrées².

Ce type de «reportage» sur l'état du judaïsme en Algérie que l'on trouvait dans la presse juive française de l'époque fit prendre conscience

1. Cité dans Michel Abitbol, «La rencontre des Juifs de France avec le judaïsme d'Afrique du Nord», Actes du colloque sur les relations intercommunautaires juives en Méditerranée occidentale XIII - XXe siècle, Paris, Éditions du CNRS, 1984, p. 229-242.

2. «Lettre d'un médecin israélite de l'Armée d'Afrique», dans Archives israélites, VI, mai 1840. 
L'émancipation à «marche forcée » : les Juifs d'Algérie...

de la profondeur du fossé culturel séparant les Juifs des deux rives de la Méditerranée. Toutefois, les autorités consistoriales restèrent prudentes face à ce discours dépréciateur et cherchèrent à s'informer par ellesmêmes. Dès 1842, elles se joignirent au ministère de la Guerre pour envoyer officiellement en Algérie une mission d'enquête sur le sujet.

Le rapport final remis au ministre de la Guerre en 1843 était signé par deux notables israélites méridionaux: le marseillais Jacques-Isaac Altaras et l'avocat aixois Joseph Cohen ${ }^{1}$. Ces derniers avaient effectué un tour presque complet de la nouvelle colonie et visité un grand nombre de communautés juives indigènes. Leur description de la situation laissait apparaître un double langage. Tout en méprisant l'ensemble des coutumes judéo-maghrébines, ils se refusaient à considérer les populations juives locales comme inférieures. Ils affirmaient même voir en elles une grande aptitude à entrer dans la voie de la civilisation. Dans ce but, ils proposèrent donc une série de mesures concrètes pour élever «l'état moral des Juifs indigènes».

On peut considérer que ce rapport fut le point de départ d'une collaboration étroite entre les autorités israélites françaises et l'État colonial. Les Juifs épris de reconnaissance pour la nation qui les a élevés au rang de citoyens trouvaient un moyen d'exprimer leur gratitude et leur croyance dans la mission civilisatrice de la France, et les autorités françaises trouvèrent un allié précieux pour mener à bien cette mission.

Le projet d'émancipation s'élabora progressivement et fut dès le départ conçu comme un processus autoritaire et centralisé. Mais contrairement à l'expérience des Juifs de France pendant la Révolution, l'octroi de droits civiques ne fut pas la première étape, il fut précédé par une série de réformes institutionnelles et la mise en place d'une tutelle solide.

\section{«Ils n'abdiqueront pas la loi de Dieu² !»}

La plus importante des mesures à apporter à l'organisation des communautés juives algériennes semblait être aux yeux de Cohen et Altaras la création de consistoires dans les grandes villes du littoral. Cette idée,

1. Ce rapport a été publié en intégralité dans Simon Schwarzfuchs, Les Juifs d'Algérie et la France (1830-1855), Jérusalem, Institut Ben-Zvi, 1981.

2. Discours d'Adolphe Crémieux cité par Charles-André Julien, Histoire de l'Algérie contemporaine: la Conquête et les débuts de la colonisation (1827-1871), Paris, PUF, 1964. 
dont on a vu plus haut les origines, était particulièrement développée dans le rapport et s'inscrivait cette fois dans un véritable projet idéologique. En effet, il s'agissait de reproduire en Algérie les mêmes schémas de modernisation religieuse qu'avait connus le judaïsme français durant la période révolutionnaire et napoléonienne. Créer des consistoires semblait être ainsi un préambule à la «régénération morale» à venir. L'objectif premier était de tracer une frontière étanche entre l'appartenance religieuse et l'appartenance civile, comme cela avait été le cas lors de l'émancipation des Juifs de France moins d'un demisiècle auparavant. Les deux auteurs du document ne faisaient que reprendre là un thème classique du discours officiel du judaïsme consistorial français qui condamnait tout espèce de regroupement corporatif ou communautaire. Pour eux, le religieux devait être absolument relégué dans la sphère privée.

En outre, la création de consistoires permettrait d'atteindre deux autres objectifs : d'une part, soustraire les communautés à l'autorité des rabbins considérés comme ferment du fanatisme en confiant la direction à une élite laïque et «éclairée»; d'autre part, casser la structure clanique de la société indigène en imposant une autorité unique.

La mise en place de consistoires était donc nécessaire, pour Cohen et Altaras, mais non suffisante. La population juive indigène étant par nature «immature», il convenait aussi de la mettre sous tutelle et de la diriger fermement depuis la métropole. D'où l'insistance particulière des auteurs sur la nécessité d'envoyer de France des grands rabbins formés à l'école de Metz et mandatés par le consistoire central pour avoir la haute main sur les activités des consistoires algériens. La mesure institutionnelle préconisée par le rapport Altaras-Cohen avait par conséquent comme ambition l'installation d'un véritable patronage: les autorités du judaïsme français étant appelées à devenir l'intermédiaire incontournable entre l'État colonisateur et les Juifs indigènes «à civiliser».

L'État monarchique français, en butte aux pressions d'un véritable lobby consistorial, finit par accepter cette solution qui jouait également dans le sens de ses intérêts. Le 9 novembre 1845, le roi signait finalement une ordonnance créant quatre consistoires en Algérie dans chaque grande ville: Alger, Oran et Constantine et un consistoire de tutelle pour l'ensemble de la colonie (lequel est supprimé vingt ans plus tard). Les consistoires régionaux prirent leurs fonctions à la fin de l'année 1847. 


\section{L'émancipation à «marche forcée »: les Juifs d'Algérie...}

L'installation de consistoires constituait en fin de compte une «révolution silencieuse » pour le judaïsme algérien, puisqu'elle avait pour but de saper en profondeur les fondements de la société judéo-maghrébine. De fait, le colonisateur introduisit par ce biais trois grandes nouveautés:

- la notion de culte public dirigé par une instance unique ayant monopole sur les activités religieuses;

- une hiérarchie: chaque communauté jusque-là indépendante devait dépendre des institutions nationales du judaïsme français;

- la distinction entre laïcs et ecclésiastiques en faisant intervenir les grands rabbins métropolitains.

Avec cette première grande réforme institutionnelle commençait aussi la campagne des Juifs de France pour la naturalisation des Juifs d'Algérie.

Cette campagne prenait sa source dans le rapport Altaras-Cohen: les deux auteurs l'évoquaient déjà comme une possibilité pour conduire à son terme le processus de «régénération» des Juifs algériens. Cependant, les autorités israélites françaises restèrent prudentes dans un premier temps. Ce n'est qu'au cours des années 1850-1860 que le lobby consistorial se remit en action auprès du gouvernement de l'Empire assez disposé à mener une telle opération.

Napoléon III lui-même donna une première grande satisfaction aux Juifs de France au cours de son voyage en Algérie. Sa visite à Oran fut l'occasion de prononcer devant le grand rabbin de la ville une phrase explicite à ce sujet: «J'espère que bientôt les Israélites d'Algérie seront citoyens ${ }^{1}$.» De fait, il promulgua à son retour à Paris en 1865 un sénatusconsulte qui autorisait les indigènes juifs et musulmans à demander à titre individuel la citoyenneté pleine et entière. Toutefois, l'opération relativement libérale tourna à l'échec puisque très peu d'indigènes firent la démarche. Dans la province d'Oran, où les Juifs étaient les plus nombreux, ce furent uniquement 192 chefs de familles qui se signalèrent dont 113 étaient d'ailleurs des immigrés récents venus du Maroc ou de Tunisie ${ }^{2}$. L'étape de la naturalisation volontaire offrit par conséquent bien des désillusions aux israélites français. Le refus des Juifs indigènes fut proportionnellement presque aussi massif que celui

1. Cité par Richard Ayoun, Typologie d'une carrière rabbinique: Mahir Charleville (1864-1877), Paris, Presses universitaires de Nancy, 1993.

2. Michel Abitbol, «La citoyenneté imposée: du décret Crémieux à la guerre d'Algérie», dans Pierre Birnbaum (dir.), Histoire politique..., op. cit. 
des musulmans. En effet, comme pour ces derniers le renoncement au statut personnel qu'impliquait la démarche signifiait une véritable apostasie, l'abandon de la loi de Dieu. Et en dehors d'une élite bourgeoise précocement francisée, l'immense majorité de la population juive ne pouvait se résoudre à accepter un tel reniement ${ }^{1}$.

Face à ce premier échec, les Juifs de France choisirent alors une option plus autoritaire pour parvenir à leurs fins, c'est-à-dire pour la naturalisation collective par décret. Ce fut le sens de la phrase qu'Adolphe Crémieux prononça à la veille de la promulgation de son décret: « $\mathrm{Ne}$ leur dites pas devenez français si vous le voulez, ils n'abdiqueront pas la loi de Dieu.»

Finalement le décret de naturalisation d'octobre 1870 fut une mesure élaborée par le cercle dirigeant du judaïsme français. Il ne fut en rien la consécration d'un état de fait - à savoir la francisation spontanée des Juifs algériens -; mais une mesure d'encouragement à entrer (de gré ou de force!) dans la normalité française.

Désormais, plus rien ne différenciait du point de vue juridique l'israélite de métropole de celui d'Algérie. Cependant les promoteurs de la civilisation eurent bien conscience de la portée du décret Crémieux et allaient redoubler d'efforts pour que les nouveaux citoyens soient dignes de leur titre. La période qui commença en 1870 et s'acheva aux lendemains de la Première Guerre mondiale aboutit certes à une intégration finale mais à la suite d'une longue série de conflits.

\section{«Soyez Français ${ }^{9}$ »}

Avec la naturalisation collective, la tutelle imposée par les Juifs de France aux Juifs algériens, loin de disparaître, se fit encore plus pesante. En effet, ce qui était acceptable pour des Juifs indigènes ne le devenait

1. On peut noter que le refus des indigènes juifs et musulmans d'abandonner leur statut personnel s'exprime auprès de l'administration française à peu près dans les mêmes termes. Il s'agit de réaffirmer pour les uns et les autres l'autorité du beit-din (tribunal rabbinique) ou du qadi (juge musulman). Sur le terrain, rien ne justifiait à ce moment-là un traitement différent pour les deux communautés, si ce n'est le découpage administratif de l'Algérie. Les Juifs étant concentrés dans les zones urbaines et littorales, ils sont dans leur écrasante majorité soumis à un pouvoir civil tandis que les populations de l'intérieur - essentiellement musulmanes - sont encore sous occupation militaire.

2. Exclamation d'un haut fonctionnaire français au sujet des responsables consistoriaux algériens. Lettre du secrétaire de la préfecture d'Oran au Gouverneur général datée du 17 juin 1905, Archives d'outre-mer AOM ALG Oran 3U27. 
plus pour des citoyens français dignes de ce nom. Il s'ensuivit donc dans le dernier quart du XIX ${ }^{\mathrm{e}}$ siècle, une ingérence plus directe des rabbins métropolitains dans les affaires communautaires et par conséquent un affrontement avec les principaux responsables des consistoires locaux.

L'exemple d'Oran est à cet égard éclairant et symptomatique. Cette période fut dominée par la puissante figure de Simon Kanouï. Ce personnage dont l'intelligence politique égalait l'opportunisme réussit la prouesse de demeurer président du consistoire quarante ans d'affilée de 1876 à 1916. Kanouï prit le contrôle de l'organisation à un moment où depuis une dizaine d'années, on procédait à une élection démocratique du président. Il fut constamment réélu à la quasi-unanimité car il bénéficiait d'un véritable soutien populaire. Fort de cette légitimité, il allait se poser en défenseur du Juif indigène et de ses traditions face à l'autorité civilisatrice symbolisée par le grand rabbin métropolitain.

À plusieurs reprises, des rabbins eurent à subir les foudres de ce redoutable adversaire politique ${ }^{1}$. Sur quatre grands rabbins en poste à Oran de 1864 à 1905, deux ne purent effectuer leur mandat jusqu'au bout et demandèrent leur rappel en métropole. Ces deux rabbins, qui, imbus de leur culture occidentale, voulurent accélérer le processus de francisation en s'imposant comme guide spirituel unique, finirent bien par porter atteinte au prestige du chef de communauté. Or, à de multiples égards, le président du consistoire - légalement investi - incarnait la continuité de l'autorité de l'ancien dignitaire maghrébin du muqaddam: c'était un personnage riche, rompu à la guerre des clans et suffisamment rusé pour triompher de ses ennemis. La lutte avec des rabbins ne disposant que de maigres réseaux d'influences s'avérait donc particulièrement inégale. Les deux grands rabbins qui purent se maintenir avaient, eux, compris et admis la prééminence de Kanouï sur la communauté. Tout en exhortant les fidèles à se vêtir à l'européenne, à abandonner le dialecte judéo-arabe, ils procédèrent par étapes, timidement, en cherchant le consensus.

De 1870 au début du $\mathrm{XX}^{\mathrm{e}}$ siècle s'installa une période de résistance passive à l'autorité consistoriale centrale et de décalage prononcé entre le culte officiel et la réalité de la vie religieuse.

1. Geneviève Dermenjian-Hannequart, «Les relations entre le consistoire central, les grands rabbins et les Juifs d'Algérie à la fin du XIXe siècle : l'exemple d'Oran (1864-1905)», Actes du colloque..., op. cit., p. 170-179. 
Certes la naturalisation avait fait progresser l'intégration républicaine grâce à la scolarisation obligatoire, mais un certain nombre de facteurs - au-delà de la pauvreté extrême et de l'illettrisme - contribuaient à la survivance du cadre communautaire ancien.

Tout d'abord, il faut mentionner l'attitude quelque peu ambiguë de l'État colonial. Officiellement rien ne devait plus distinguer les israélites d'Algérie de leurs homologues de métropole. Or, dans certains cas - certes très limités - les Juifs algériens étaient renvoyés à leur ancienne condition d' «indigènes ». Ce fut le cas par exemple en 1892, lorsqu'un décret réorganisa l'enseignement religieux traditionnel. Écoles coraniques et écoles talmudiques étaient placées sous la même autorité et soumises aux mêmes règles. La reconnaissance officielle des midrashim (écoles traditionnelles d'enseignement judéo-arabe) ruinait une partie des efforts des rabbins métropolitains chargés de réformer l'éducation religieuse.

Un deuxième facteur jouait contre l'action des rabbins consistoriaux: la puissance des réseaux familiaux. La majeure partie des lieux de cultes demeurèrent pendant cette période des espaces privés sous l'autorité d'un chef de clan. En 1900, on dénombrait à Alger (avec la synagogue Randon par exemple) ou à Oran seulement un ou deux édifices publics de culte pour des populations de plus de 10000 fidèles. Les dizaines d'oratoires privés recensés dans ces villes n'étaient ouverts qu'à une clientèle réduite, échappant totalement à l'emprise du consistoire et de ses représentants ${ }^{1}$.

Enfin, le troisième frein à l'assimilation au judaïsme français était l'influence des rabbins traditionnels. A priori, l'une des prérogatives essentielles de l'organisation consistoriale était la désignation des ministres du culte. Or, la séparation entre laïcs et religieux ne trouvait aucun écho dans une société où le rabbinat était une institution sociale multiforme. À la fois enseignants, officiants, sacrificateurs et quêteurs, les rabbins traditionnels pour la plupart auto-désignés conservaient un rôle majeur dans le maintien des coutumes ancestrales dans les familles ${ }^{2}$. Le prestige de l'autorité rabbinique se remarquait aussi par la permanence d'un dayan (ancien dignitaire du tribunal rabbinique) dans

1. C'est ce qui est observé dans L'enquête générale sur la situation du culte et de la bienfaisance en Algérie par le grand rabbin Isaac Bloch, document confidentiel dactylographié non daté (1909), Archives du consistoire central, non classé.

2. David Nadjari, Juifs en terre coloniale, Nice, Gandini, 2000. 
L'émancipation à «marche forcée » : les Juifs d'Algérie...

la plupart des communautés, consultés y compris par les membres des consistoires !

Au début du $\mathrm{XX}^{\mathrm{e}}$ siècle, la francisation de la société juive algérienne était donc certes en marche sous les effets conjugués de la scolarisation et de l'administration consistoriale, mais elle rencontrait de fortes résistances sur le terrain.

La loi de séparation des Églises et de l'État de 1905 intervint dans ce contexte et remit sérieusement en cause la tutelle exercée par les Juifs de France sur leurs «frères» d'Algérie.

Au moment de sa discussion en métropole, la loi de séparation des cultes et de l'État fit naître une inquiétude chez les notables israélites français. S'il était appliqué en Algérie, le nouveau régime des cultes aurait pour conséquence la création d'associations cultuelles libres et donc la fin du contrôle étroit de la métropole sur les consistoires locaux ${ }^{1}$.

Après avoir été reportée, l'application du nouveau statut des Églises fut finalement votée en 1908. Mais la nouvelle législation n'eut guère les effets redoutés. Certes, immédiatement des dizaines d'associations cultuelles dissidentes virent le jour dans les grandes villes algériennes, mais dans la plupart des cas, seulement une ou deux purent prétendre au statut de consistoire. Les autres retournant au cadre familial. L'unité du judaïsme algérien ne fut pas brisée grâce à l'accaparement des consistoires par l'élite ploutocratique algérienne. Ils eurent tout intérêt à garder l'institution cultuelle sous sa forme centralisée pour mieux asseoir leur pouvoir sur le reste de la communauté.

C'est donc naturellement que les associations cultuelles algériennes adhérèrent dès 1909 à la Fédération des consistoires de France pour conserver un instrument administratif indispensable vis-à-vis de l'autorité coloniale.

Cette période fut aussi marquée par un tournant important dans les relations entre Juifs de France et Juifs d'Algérie.

Les premiers trouvèrent peu à peu des alliés dans la société algérienne: la nouvelle génération de notables formée depuis 1870 à l'école républicaine. À l'émancipation «par le haut» succéda une nouvelle émancipation «par le bas».

1. Simon Schwarzfuchs, «Le judaïsme algérien devant le choix: la crise de 1905 », Actes du colloque..., op. cit. 
Dans la plupart des consistoires départementaux, on assista à une lutte de pouvoir entre les élites communautaires traditionalistes et une nouvelle génération éclairée. La rivalité entre les clans familiaux épousait de plus en plus le clivage entre modernistes et traditionalistes. Dans la province d'Oran, la plus peuplée par les Juifs, la «chute» de Simon Kanouï en $1915^{1}$ ouvrit la voie à une recomposition totale du paysage politique communautaire. Au lendemain de la Première Guerre mondiale, le personnel consistorial fut en grande partie renouvelé, et le rapport de force tourna à l'avantage des chefs de clans «modernistes». À Alger et Oran, la période d'affrontement entre rabbins métropolitains et dirigeants communautaires trouva aussi une issue partielle dans la nomination de grands rabbins d'origine algérienne mais formés au séminaire israélite français à Paris².

Les autorités consistoriales devinrent donc plus souples dans leurs rapports avec les consistoires d'outre-mer, d'autant plus que l'Alliance israélite universelle, absente jusqu'alors d'Algérie ${ }^{3}$, commence son action d'éducation en ouvrant entre 1900 et 1907 des écoles dans les trois grandes villes algériennes. Le rôle de cette organisation missionnaire est significatif en Algérie. Sans se substituer à l'école républicaine, l'AIU entend réduire l'influence des rabbins judéo-arabes en proposant une formation technique et professionnelle assortie d'un «catéchisme ${ }^{4}$ » israélite en français! On dénombre plus de deux cents élèves dans l'école d'Oran en 1908 dont une petite moitié est constituée de filles, exclues de fait des midrashim traditionnels.

Au moment où les conscrits d'Algérie répondent à l'appel patriotique de la Grande Guerre, on peut dire que le processus d'émancipation politique des Juifs algériens est achevé. La méthode autoritaire de l'État secondé par les autorités israélites françaises a porté ses fruits au moins sur le plan officiel, plus rien ne distingue un consistoire algérien

1. Simon Kanoui meurt en 1915 sans successeur désigné. Voir David Nadjari, «Simon Kanouï (18421915)», dans Archives juives, 36/1, 2003, p. 136-139.

2. C'est notamment le cas du rabbin David Ashkenazi, en poste à Oran dès les années 1920.

3. Jusqu'au début du $\mathrm{XX}^{\mathrm{e}}$ siècle, et contrairement au reste du bassin méditerranéen, l'Alliance israélite universelle ne considérait pas l'Algérie comme une terre de «mission», mais comme un ensemble de départements assimilés au territoire national. Dès lors, la mission d'instruction revenait exclusivement à l'école républicaine.

4. «Catéchisme» est le mot employé dans tous les documents officiels pour qualifier l'histoire sainte et l'instruction religieuse, matières principales enseignées dans ces écoles. Voir Emploi du temps des classes de filles et garçons, mai 1908, Archives de l'Alliance israélite universelle AIU VE 48. 
L'émancipation à «marche forcée » : les Juifs d'Algérie...

de ses homologues de métropole, et les Juifs sont des citoyens jouissant de tous leurs droits civiques.

Cependant, près d'un demi-siècle après leur naturalisation collective, la réforme du culte, corollaire de l'émancipation, en dépit d'incontestables progrès, est toujours virtuelle dans une partie de la société juive. Le franco-judaïsme, forme modernisée du culte israélite né de l'époque napoléonienne, n'a de place que dans quelques synagogues publiques contrôlées par les consistoires et dans les écoles de l'Alliance israélite universelle. En dépit de la progression du français comme langue courante, la liturgie, les rites traditionnels judéo-arabes survivent dans le cercle familial.

La communauté comme corps politique a disparu, mais sa substance sociale reste encore largement active, notamment grâce à la survivance de clans familiaux puissants.

La résistance «culturelle» au franco-judaïsme des Juifs algériens est une réalité qui va demeurer sous des formes de plus en plus souples jusqu'au rapatriement de 1962. Ironie de l'histoire, ce sont ceux qui se sont vus imposer le modèle consistorial qui vont réintroduire en France - avec tous les autres Juifs d'Afrique du Nord - le modèle communautaire. 